\title{
Thalamus, Brainstem and Salience Network Connectivity Changes During Propofol-Induced Sedation and Unconsciousness
}

\author{
Pieter Guldenmund, Athena Demertzi, ${ }^{1,2}$ Pierre Boveroux, ${ }^{1,3}$ Mélanie Boly, ${ }^{1,2}$ Audrey Vanhaudenhuyse, ${ }^{1,2}$ \\ Marie-Aurélie Bruno,, ${ }^{1,2}$ Olivia Gosseries, ${ }^{1,2}$ Quentin Noirhomme,, Jean-François Brichant, ${ }^{3}$ \\ Vincent Bonhomme, ${ }^{1,3,4}$ Steven Laureys, ${ }^{1,2}$ and Andrea Soddu ${ }^{5}$
}

\begin{abstract}
In this functional magnetic resonance imaging study, we examined the effect of mild propofol sedation and propofol-induced unconsciousness on resting state brain connectivity, using graph analysis based on independent component analysis and a classical seed-based analysis. Contrary to previous propofol research, which mainly emphasized the importance of connectivity in the default mode network (DMN) and external control network (ECN), we focused on the salience network, thalamus, and brainstem. The importance of these brain regions in brain arousal and organization merits a more detailed examination of their connectivity response to propofol. We found that the salience network disintegrated during propofol-induced unconsciousness. The thalamus decreased connectivity with the DMN, ECN, and salience network, while increasing connectivity with sensorimotor and auditory/insular cortices. Brainstem regions disconnected from the DMN with unconsciousness, while the pontine tegmental area increased connectivity with the insulae during mild sedation. These findings illustrate that loss of consciousness is associated with a wide variety of decreases and increases of both cortical and subcortical connectivity. It furthermore stresses the necessity of also examining resting state connectivity in networks representing arousal, not only those associated with awareness.
\end{abstract}

Key words: consciousness; pons; propofol; resting state fMRI; salience network; thalamus

\section{Introduction}

C ONSCIOUSNESS Is a phenomenon that has so far evaded detailed description. With the advent of modern brain imaging techniques, it has become possible to better locate changes in brain activity associated with altered consciousness. In the healthy awake brain, structured brain activity occurs even in the absence of a specific task. This spontaneous activity is organized into resting state networks (RSNs) (Damoiseaux et al., 2006). Each RSN consists of brain regions that have a comparable temporal pattern of spontaneous neuronal activity, which are said to be functionally connected. Resting state functional magnetic resonance imaging (resting state fMRI) is an established method used to examine changes in the brain's connectivity when consciousness is altered, as- suming that low frequency changes in blood oxygenation reflect neuronal activity. It has been demonstrated that especially higher-order RSNs show decreases in connectivity when consciousness is reduced or lost. The default mode network (DMN), external control network (ECN), and salience network are considered to represent the major portion of higher-order RSNs. The DMN is known to show changes in the spatial pattern during altered states of consciousness like deep sleep, vegetative state, and general anesthesia (Boveroux et al., 2010; Guldenmund et al., 2012; Horovitz et al., 2009; Schrouff et al., 2011; Vanhaudenhuyse et al., 2009). More recent studies also show substantial disintegration of the ECN, an RSN with spontaneous neuronal activity that is anticorrelated to that of the DMN (Boveroux et al., 2010; Vanhaudenhuyse et al., 2011), with decreasing

\footnotetext{
${ }^{1}$ Coma Science Group, Cyclotron Research Center, University of Liège, Liège, Belgium.

Departments of ${ }^{2}$ Neurology and ${ }^{3}$ Anesthesia and Intensive Care Medicine, CHU Sart Tilman Hospital, University of Liège, Liège, Belgium.

${ }^{4}$ Department of Anesthesia and Intensive Care Medicine, CHR Citadelle, University of Liège, Liège, Belgium.

${ }^{5}$ Physics \& Astronomy Department, Mind \& Brain Institute, Western University, London, Canada.
} 
consciousness. However, although the DMN and ECN are associated with the mediation of internal and external awareness, respectively, and are thus logical networks of interest for anesthesia studies, these are not the only brain networks showing changes during loss of consciousness. For instance, little is known about connectivity of the salience network during mild sedation and unconsciousness. The salience network is an RSN that consists of the bilateral frontal insulae, anterior cingulate cortex, and presupplementary motor area (Seeley et al., 2007). Activity in the salience RSN is associated with the detection of novel, salient stimuli, and is thought to play a role in coordinating an adequate response by recruiting appropriate brain networks. Therefore, some authors suggest it might play a role in coordinating between DMN and ECN activity (Bonnelle et al., 2012; Menon and Uddin, 2010).

Furthermore, although some resting state fMRI literature discusses changing connectivity between higher-order, highly integrative networks, and the thalamus, much remains to be discovered about changing connectivity between thalamus, brainstem and lower-order networks (like the auditory and sensorimotor RSNs). The propofol study by Boveroux et al. (2010) showed an involvement of the thalamus and brainstem regions in propofol-induced unconsciousness. However, no separate seed-based analysis with these loci as seed regions was performed to document their connectivity changes in detail. The brainstem pontine tegmental area (PTA), mesencephalon, and thalamus are brain structures closely associated with brain arousal via the ascending reticular arousal system (Angeles Fernandez-Gil et al., 2010; Giacino et al., 2012). In fact, microinjection of pentobarbital in the mesopontine tegmental region in rats has been shown to result in a condition resembling general anesthesia (Devor and Zalkind, 2001). Furthermore, the thalamus is regarded as one of the key loci for information integration and brain RSN coordination (Brown et al., 2010; Pinault, 2011; Schiff, 2008; Schiff and Fins, 2007; Tang et al., 2011). In general, propofol-induced unconsciousness is thought to result from increased GABA-ergic transmission in the cortex and at inhibitory projections from the preoptic area of the hypothalamus to arousal regions in the brainstem (Brown et al., 2011). Physiological effects of propofol, such as atonia and apnea, can also be traced back to the drug's actions on the brainstem (Brown et al., 2011).

These findings stress the potential importance of brainstem, thalamus and salience RSN activity in propofolinduced mild sedation and loss of consciousness. Therefore, we further examined their functional connectivity with resting state fMRI. Potential thalamic and brainstem involvement in the DMN and auditory RSN was first tested using a recently developed analysis method based on independent component analysis (ICA). Next, we used a classical seedbased technique to further examine connectivity changes with regions in the salience network, thalamus, and brainstem. A seed was also placed in the hippocampus, to examine expected changes in connectivity with the DMN other than the previously described frontoparietal disconnection (Boveroux et al., 2010). Given its role in the detection of salient stimuli and thus direction of attention, we expected decreases in salience network connectivity with unconsciousness, while the PTA, mesencephalon and thalamus, being involved in brain arousal and information integration, are also likely to show a propofol-induced change in connectivity.

\section{Materials and Methods}

\section{Subjects}

We used previously published fMRI data from 20 healthy right-handed volunteers (Boveroux et al., 2010; Schrouff et al., 2011). One subject was excluded from the analysis due to the occurrence of hyperventilation, while data from two other subjects were left out as they were acquired during pilot sessions. Data from the remaining 17 volunteers (13 women and 4 men; mean age: 21.9 years; standard deviation: 1.9 years; none was under medication or pregnant) were used for our analysis. The study was approved by the Ethics Committee of the Faculty of Medicine of the University of Liège (University Hospital, Liège, Belgium) and subjects gave written informed consent.

\section{Sedation protocol}

Subjects fasted for at least $6 \mathrm{~h}$ for solids and $2 \mathrm{~h}$ for liquids before the sedation. They wore headphones and earplugs in the scanner. Propofol infusion, using a target controlled infusion device (Diprifusor $(C-$-algorithm, Pharmacokinetics and Pharmacodynamics Software Server, Department of Anesthesia, Stanford University, USA) to obtain constant effect-site concentrations, occurred via an intravenous catheter placed into a vein of the right forearm or hand. During all four levels of consciousness, the blood pressure, electrocardiogram, breathing frequency, and pulse oxymetry $\left(\mathrm{SpO}_{2}\right)$ were continuously monitored. For the whole duration of the experiment, subjects were breathing spontaneously, while additional oxygen was delivered at $5 \mathrm{~L} / \mathrm{min}$ via a loosely fitting plastic facemask. The level of consciousness was assessed using the Ramsay scale (Olson et al., 2007). The subject was asked twice per consciousness level assessment to strongly squeeze the hand of the investigator. The awake states before sedation and after recovery of consciousness were Ramsay 2 (strong squeezing of the hand), mild sedation was Ramsay 3 (clear but slow squeezing), and propofol-induced unconsciousness was Ramsay 5-6 (no response). In addition, a reaction time task was also given to the subject before and after each session to help define the level of consciousness. This reaction task consisted of a block of 20 beeps delivered via the headphones, and the subjects were asked to press a keypad as fast as they could. After reaching the desired effect-site concentration, a $5 \mathrm{~min}$ equilibration period was established. Mean propofol plasma concentrations for wakefulness, mild sedation, unconsciousness, and recovery were $0 \mu \mathrm{g} / \mathrm{mL}$ (standard deviation: 0 ), $1.71 \mu \mathrm{g} / \mathrm{mL}$ (standard deviation: 0.72 ), $3.02 \mu \mathrm{g} / \mathrm{mL}$ (standard deviation: 1.03 ), and $0.59 \mu \mathrm{g} / \mathrm{mL}$ (standard deviation: 0.28 ), respectively. These propofol measurements were based on arterial blood samples taken directly before and after each scan. Two certified anesthesiologists and complete resuscitation equipment were present throughout the experiment [for supplementary protocol information, see Boveroux et al. (2010)].

\section{fMRI data acquisition}

Functional images were acquired on a 3T Siemens Allegra scanner (Siemens AG, Munich, Germany; Echo Planar Imaging sequence using 32 slices, repetition time $=2460 \mathrm{~ms}$, echo time $=40 \mathrm{~ms}$, field of view $=220 \mathrm{~mm}$, voxel size $=3.45 \times 3.45 \times$ $3 \mathrm{~mm}$, matrix size $=64 \times 64 \times 32$ ). Ten minute acquisitions were made (mean: 253 scans, standard deviation: 74 scans) 
during four different states of consciousness: normal wakefulness, mild propofol sedation, propofol-induced unconsciousness, and recovery. The temporal order of mild sedation and propofol-induced unconsciousness sessions was randomized. A high-resolution T1 image was also made for each subject for coregistration purposes. Total scan time per condition was around $30 \mathrm{~min}$.

\section{ICA-based analysis}

The first analysis method we used was an automated ICAbased process combined with graph theory. This approach was used to analyze propofol-induced connectivity changes of whole networks: the DMN and the auditory RSN. These RSNs were chosen as they, and regions anticorrelating with the DMN and auditory RSN, are well-described and robust. This is important for the selection of regions of interest (ROIs) representing the RSNs, a vital part of our selection procedure as will be described below. Furthermore, these two RSNs are thought to be good representatives of a "higherorder," highly integrative RSN and a sensory "lower-order" RSN, respectively. Structural and functional magnetic resonance images were realigned, normalized, smoothed $(8 \mathrm{~mm}$ kernel), and corrected for 3D motion using BrainVoyager QX software (R. Goebel, Brain Innovation, Maastricht, The Netherlands). ICA with 30 components yielded statistically independent RSNs and possible artifacts (Hyvarinen et al., 2001; McKeown et al., 1998). Independent components corresponding to the DMN were selected using a previously published automated selection technique (Maudoux et al., 2012; Soddu et al., 2012). For this technique, using an average DMN template calculated on the basis of resting state fMRI scans from 12 healthy subjects [ 4 women, 8 men; mean age 21 years; standard deviation: 3 years (Demertzi et al., 2011)], who were scanned for a previous study on a 3T Siemens Allegra scanner (10 min resting state with eyes closed), we defined 14 ROIs $(10 \times 10 \times 10 \mathrm{~mm})$ that were considered to be most representative of the DMN based on previous research (Fair et al., 2008; Fox et al., 2005) (Supplementary Material S1; Supplementary Data are available online at www.liebertpub.com/brain). These ROIs were employed in the automatic selection process to choose the component that showed the highest level of total connectivity between these nodes, thereby being most likely to be the DMN component. A connectivity graph was created for each of the 30 independent components produced by ICA. For a given graph, edges between each pair of ROIs represented how the corresponding time course was predicting the blood oxygenation level dependent (BOLD) signal in that pair. To be certain that the global signal was not chosen as the best representative of the DMN, a method was devised to remove the independent component representing the global signal from the automatic selection process (Soddu et al., 2012). For this, beta values from regions that are known to anticorrelate to the DMN were introduced as a weight. The value of this weight was dependent on the amount of anticorrelation with the regions that are known to anticorrelate with the $\mathrm{DMN}$, becoming stronger when less anticorrelation is present. As the global signal does not anticorrelate with these regions and the DMN does, this made sure the independent component representing the DMN was selected rather than the global signal. To ensure that neuronal independent com- ponents were selected rather than artifacts, temporal characteristics of the independent components were added to the selection process, by comparing the components to an average component fingerprint [obtained from 12 controls scanned for a previous study (Demertzi et al., 2011)] (De Martino et al., 2007). This fingerprint is a collection of characteristics of each independent component, including information about frequency behavior, clustering, skewness, kurtosis, spatial entropy, one lag autocorrelation, and temporal entropy. Specific values of these characteristics imply that a component is truly neuronal (De Martino et al., 2007). The automated selection process used these values to favor selection of a more neuronal component fitting the spatial pattern (goodness of fit) above non-neuronal components. This process yielded one selected connectivity graph per subject per consciousness condition representing DMN connectivity. These graphs were then averaged across all subjects, resulting in one graph per condition. Connectivity graph contrasts representing connectivity differences between different consciousness conditions were constructed using the four averaged graphs. Graph connections with $p$-values that survived Bonferroni correction for the average across subjects were represented by thick lines, while uncorrected $p$-values were drawn as thin lines. In addition to the connectivity graphs, beta maps were generated by regressing the BOLD signal with the DMN time course (keeping all the other components' time courses as confounds). The obtained beta maps were subsequently used in a random effects analysis to construct a $t$-test spatial map per consciousness condition. Contrast $t$-test spatial maps were also calculated. The auditory RSN was examined in a similar way as the DMN. This well-described robust sensory network was selected to have a way of comparing the effect of propofol on the DMN with its effect on a sensory RSN. Eleven ROIs were chosen to represent the auditory RSN, based on the same 12 subjects as those used to determine DMN ROIs (Demertzi et al., 2011) (Supplementary Material S1). For illustrative purposes, we superimposed on the connectivity graphs of each condition of the DMN (Fig. 1) and auditory RSN (Fig. 2) nodes between which connectivity changed significantly between conditions as seen when performing $t$-tests (thick blue circles for decreased connectivity in the DMN and thick red circles for increased connectivity in the auditory RSN).

\section{Seed-based analysis}

Although our automatic ICA method is a robust tool for analyzing the well-described DMN and auditory RSN, seed analysis was chosen for further analysis of the connective repertoire of ROIs arising from previous research in the context of consciousness. Regions selected for the seed-based analysis were the right anterior insular cortex $(33,22,6)$, thalamus $(-7,-16,6$ and $7,-16,6$ combined), mesencephalon $(-2$, $-24,-8)$, PTA $(-3,-18,-27)$, and hippocampus $(25,-16$, $-15)$. Literature confirms strong relationships of these regions with cognition and consciousness: the PTA, mesencephalon, and thalamus are of interest because of their roles in cortex activation and coordination (Abulafia et al., 2009; Saper et al., 2005; Schiff, 2008), while the hippocampus has a pivotal role in memory processing and has a close association with the DMN, providing additional information about DMN integrity (Wang and Orser, 2011). The right anterior insular 


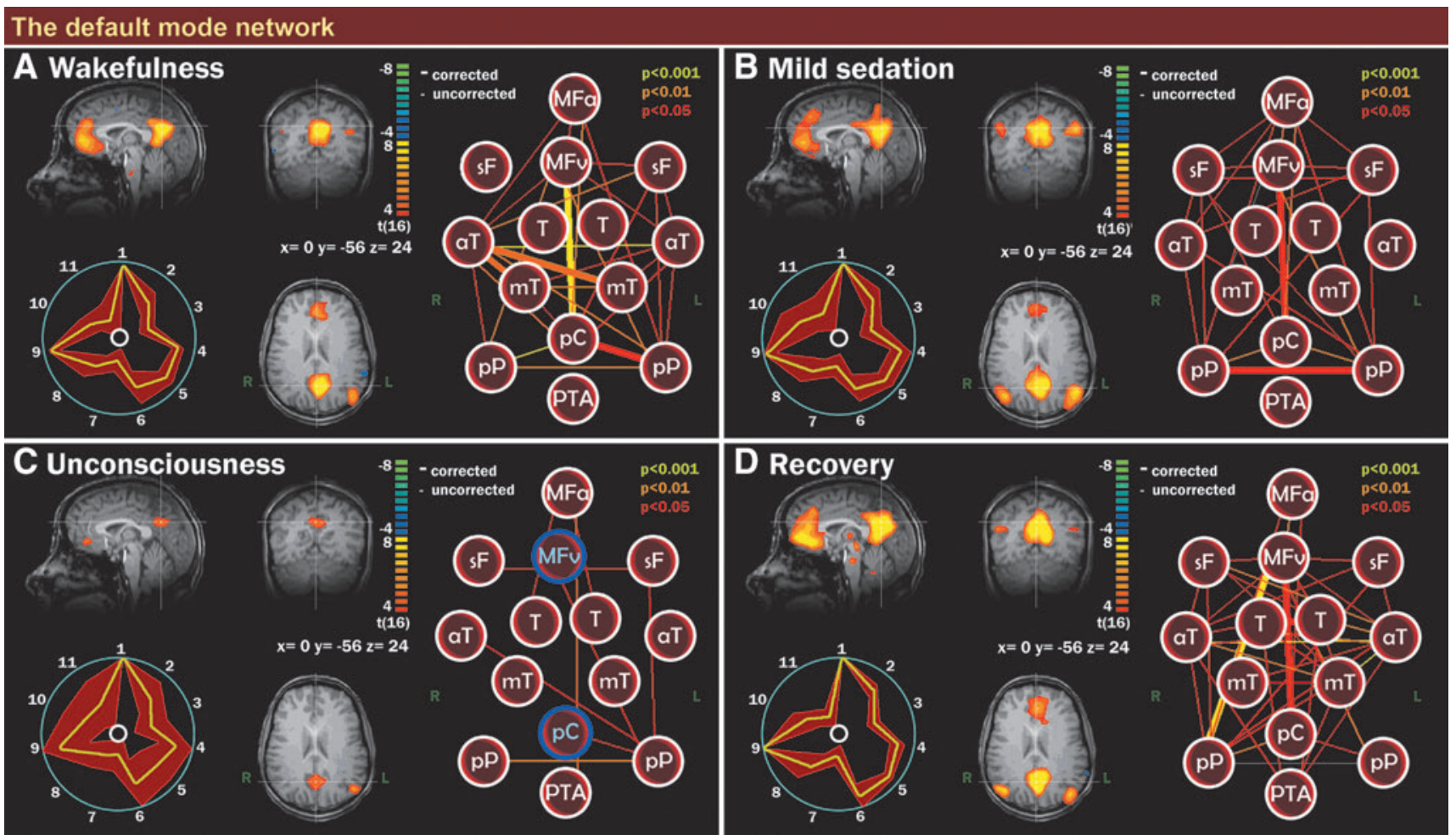

FIG. 1. Default mode network (DMN) integrity during wakefulness, mild sedation, unconsciousness, and recovery. Scalar maps (left in each panel), fingerprints (bottom left in each panel) and connectivity graphs (right in each panel) in four conditions: wakefulness (the right inferior parietal region of the DMN is just below the z-axis of the image) (A), mild sedation (B), unconsciousness (C), and recovery (D). Scalar map legend: the yellow/orange regions (yellow/orange color scale) represent DMN regions; blue/green regions (blue/green color scale) are anticorrelated to the DMN regions. $p<0.001$ uncorrected, cluster size corrected (Supplementary Material S4). Fingerprint legend: 1, Clustering; 2, Skewness; 3, Kurtosis; 4, Spatial entropy; 5, One lag autocorrelation; 6, Temporal entropy; 7, 0-0.008 Hz; 8, 0.008-0.02 Hz; 9, 0.02-0.05 Hz; 10, 0.05-0.1 Hz; 11, 0.1-0.25 Hz. Connectivity graph legend: MFa, medial frontal cortex anterior; MFv, medial frontal cortex ventral; sF, superior frontal cortex; $\mathrm{T}$, thalamus; aT, anterior temporal cortex; $\mathrm{mT}$, medial temporal/parahippocampal cortex; $\mathrm{pC}$, precuneus; $\mathrm{pP}$, posterior parietal; PTA, pontine tegmental area. R, right; L, left. Thick lines are functional connections that survive multiple comparisons. Blue-bordered circles represent DMN regions between which connectivity has dropped in that consciousness state as compared to wakefulness, based on a two-sample t-test between conditions (Supplementary Material S3).

cortex has been associated with the making of go/no-go decisions (Aron et al., 2004; Duann et al., 2009) and is part of the salience network, which is thought to enable switching between internally-oriented thought (DMN-driven) and externally-oriented activity (ECN-driven) (Seeley et al., 2007). For the same 17 subjects that we used for our ICA-based analysis, and for all four consciousness conditions, we used SPM8 software (statistical parametric mapping, Wellcome Trust Centre for Neuroimaging, www.fil.ion.ucl.ac.uk) to realign, normalize, smooth ( $8 \mathrm{~mm}$ kernel), and analyze the data. Results were thresholded at family-wise error corrected $p$ value $<0.05$ at the whole brain level or in a $10 \mathrm{~mm}$ radius spherical small volume around a priori coordinates taken from previous studies (Boly et al., 2009; Boveroux et al., 2010; Demertzi et al., 2011; Fair et al., 2008; Greicius et al., 2008; Martuzzi et al., 2010). For display purposes, data are presented at $p<0.001$ (uncorrected).

Although whole brain family-wise error correction, and to lesser extent whole brain false discovery rate correction, are often employed in studies where relatively great changes in brain activity can be expected (such as sensory and motor processes in classical fMRI studies), more subtle changes will go unnoticed. In fMRI research, there is a trend towards dramatically trying to minimize the chance of finding false positives (type I errors), resulting in an increase in type II errors (missing true effects). However, a great number of highly reproducible studies have used $p<0.001$ uncorrected thresholds (Lieberman and Cunningham, 2009). This threshold is generally considered to be a good balance between the chance of finding type I and II errors (Lieberman and Cunningham, 2009). Nevertheless, carefulness is advised with the interpretation of clusters consisting of less than 10 voxels. The employment of knowledge of the region's behavior in comparable experimental setups outlined in previous studies, used for applying small volume correction at a family-wise error of $p<0.05$ in this study, is a commonly employed procedure in the field of fMRI and further aids in minimizing potential type I errors.

\section{Results}

\section{ICA of the DMN}

During wakefulness before administration of propofol, DMN connectivity could be observed in all subjects (Fig. 

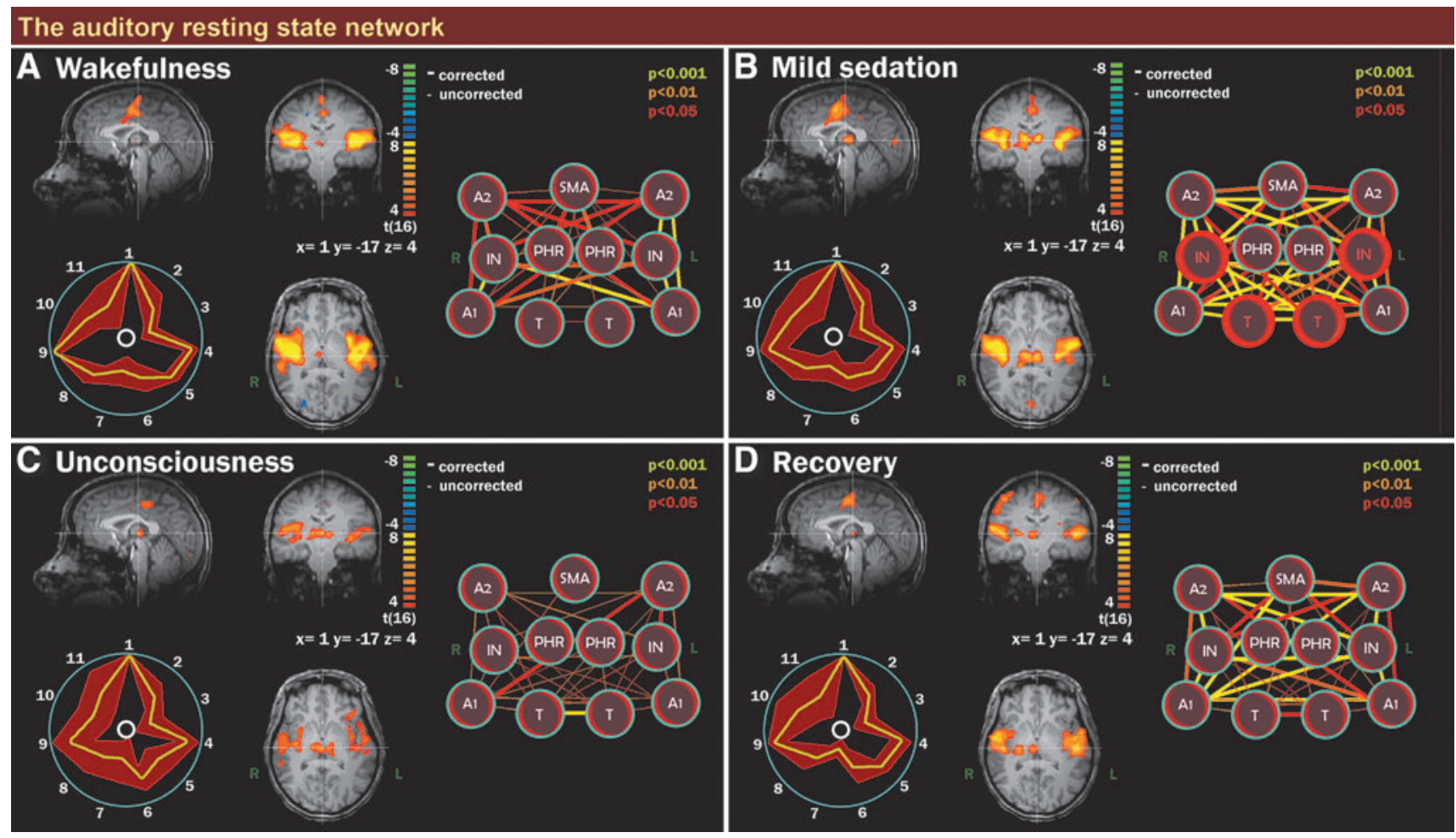

FIG. 2. Auditory resting state network (RSN) integrity during wakefulness, mild sedation, unconsciousness, and recovery. Scalar maps (left in each panel), fingerprints (bottom left in each panel), and connectivity graphs (right in each panel) in four conditions: wakefulness (A), mild sedation (B), unconsciousness (C), and recovery (D). Scalar map legend: the yellow / orange regions (yellow/orange color scale) represent auditory RSN regions; blue/green regions (blue/green color scale) are anticorrelated to the auditory RSN regions. $p<0.001$ uncorrected, clustersize corrected (Supplementary Material S4). Fingerprint legend: 1, Clustering; 2, Skewness; 3, Kurtosis; 4, Spatial entropy; 5, One lag autocorrelation; 6, Temporal entropy; 7, 0-0.008 Hz; 8, $0.008-0.02 \mathrm{~Hz} ; 9,0.02-0.05 \mathrm{~Hz} ; 10,0.05-0.1 \mathrm{~Hz} ; 11,0.1-0.25 \mathrm{~Hz}$. Connectivity graph legend: SMA, supplementary motor area; A2, secondary auditory cortex; PHR, parahippocampal region; IN, insula; A1, primary auditory cortex; T, thalamus. R, right; L, left. Thick lines are functional connections that survive multiple comparisons. Red-bordered circles represent auditory RSN regions between which connectivity has increased in that consciousness state as compared to wakefulness, based on a twosample $t$-test between conditions (Supplementary Material S3).

2A). The DMN consisted of medial prefrontal cortex, posterior cingulate cortex/precuneus, and bilateral inferior parietal cortices (Beckmann et al., 2005; Damoiseaux et al., 2006). With mild propofol sedation, no significant changes were observed in DMN connectivity strength as compared to wakefulness (Fig. 2B, see Supplementary Material S2, DMN, for contrasts). The scalar contrast map showed that anticorrelation between the bilateral anterior insular cortices and the DMN had decreased from wakefulness to mild sedation (Supplementary Material S2, DMN).

During propofol-induced unconsciousness (Fig. 2C), the only difference found with the graph analysis between wakefulness and unconsciousness that survived bonferroni multiple comparisons correction was the loss of frontoparietal connectivity (Fig. 2C \& Supplementary Material S2, DMN). Disconnection of the frontal part of the DMN was seen on the scalar map. As with mild sedation, the bilateral anterior insular cortices were less anticorrelated to the DMN as compared to wakefulness (Supplementary Material S2, DMN).

Recovery of consciousness was accompanied by the reestablishment of frontoparietal connectivity (Fig. 2D). No significant differences were observed between wakefulness before sedation and recovery, apart from weaker anticorrela- tion of the right anterior insular cortex with the DMN during recovery, as seen on the contrast scalar map (Supplementary Material S2, DMN). On the scalar map, two brainstem areas (the PTA and a region of the mesencephalon) showed up as being functionally connected to the DMN, whereby the mesencephalon demonstrated a significant increase in connectivity to the DMN during recovery from unconsciousness (Supplementary Material S2, DMN). We also found the thalamus and the cerebellum to be connected to the DMN during recovery (Fig. 2D).

The fingerprints from the chosen components of all four conditions were similar to the average fingerprint obtained from healthy, nonsedated controls (De Martino et al., 2007; Demertzi et al., 2011), and were thus likely to be neuronal. Movement parameters showed that there were no significant differences in patient movement between patients, or between scanning sessions.

\section{ICA of the auditory RSN}

The identified auditory RSN during wakefulness consisted of bilateral insular regions, the (supplementary) motor area and thalamus (Fig. 2A). An increase in connectivity between 
thalamus and insula was observed during mild sedation (Fig. 2B). No other significant differences were observed during either of the four consciousness conditions (Fig. 2C, D; Supplementary Material S2, auditory RSN). During wakefulness, we observed on the scalar maps an anticorrelation between the auditory RSN and two bilateral homotopic parts of the secondary visual cortex, while during mild sedation; connectivity was seen between the auditory RSN and a medial part of the secondary visual cortex.

\section{Seed-based analysis}

1. The first seed was placed in the anterior insular cortex (Supplementary Material S3). Decreased connectivity between the anterior insular cortex and bilateral inferior parietal cortices, precuneus, secondary sensorimotor area, and primary auditory cortex was found during mild sedation. Proceeding from mild sedation to unconsciousness, the anterior insular cortex also disconnected from the the salience network, thalamus, and superior temporal gyrus.

2. The second seed region of our analysis was the thalamus (Fig. 3 \& Supplementary Material S3). With mild sedation, an increase in connectivity between the thalamus and the auditory cortex, insular cortex, primary somatosensory cortex, primary motor cortex, and supplementary motor area was observed. Decreased connectivity was found between thalamus and cerebellum. Compared to mild sedation, unconsciousness was accompanied by a decrease in thalamic connectivity with insular regions. Connectivity between thalamus and both the salience network regions and bilateral inferior parietal lobules was found to decrease from mild sedation to unconsciousness. Thalamic connectivity with regions overlapping with those of the DMN decreased during unconsciousness as compared to wakefulness. Connectivity between thalamus and supplementary motor and primary motor areas remained stronger than during full wakefulness.

3. In the mesencephalon seed region (Supplementary Material S3), mild sedation was associated with decreased connectivity with the posterior cingulate cortex/precuneus and the mesencephalon itself. Proceeding from mild sedation to unconsciousness, the seed region decreased connectivity with the posterior cingulate cortex/precuneus and left inferior parietal cortex. Compared to wakefulness, unconsciousness was associated with decreased connectivity with the posterior cingulate cortex/precuneus, inferior parietal cortices, and anterior cingulate cortex.

4. The fourth seed region was the PTA (Fig. 4 \& Supplementary Material S3). During mild sedation, the PTA became more connected to the auditory and other insular regions. From mild sedation to unconsciousness, a decrease in connectivity compared to mild sedation was observed between PTA and auditory/insular areas, until preanesthesia levels of connectivity. Compared to wakefulness, during unconsciousness, a decrease in PTA connectivity with the posterior cingulate cortex/ precuneus and right superior frontal cortex was found, while connectivity between PTA and putamen increased. During both mild sedation and unconsciousness, a decreased connectivity between PTA and thalamus was found.

5. The fifth seed region was the hippocampus (Supplementary Material S3). With mild sedation, we found a connectivity decrease between hippocampus and both posterior cingulate cortex/precuneus and primary/secondary visual cortex. From mild sedation to unconsciousness, a decrease in connectivity between hippocampus and the inferior parietal region was observed.

\section{Discussion}

We examined the changing connectivity of the thalamus, brainstem and salience network during mild propofol sedation and propofol-induced unconsciousness. The results of this analysis complement the research performed by Boveroux et al. (2010), who focused on DMN and ECN connectivity changes associated with a decreasing level of consciousness. This previous research found propofol-induced decreased frontoparietal connectivity in the DMN, decreased anticorrelation between DMN and ECN, and decreased connectivity between thalamus and both DMN and ECN. Furthermore, they reported decreased connectivity between visual and auditory RSNs, while connectivity within the auditory and visual RSNs did not change significantly. We here present results from our analyses focusing on propofol-induced salience network, thalamus, and brainstem connectivity changes.

\section{Salience network}

Disconnection within the salience network and between salience network regions and thalamus was found with the thalamus and anterior insular cortex seeds. This disconnection has not been previously described in anesthesia and could play a major role in loss of consciousness. The influence of correct salience network functioning on brain connectivity was shown in a study with patients with cognitive impairment following mild traumatic brain injury (Bonnelle et al., 2012). The patients were performing a stop-signal task. Rapid deactivation of DMN regions was associated with efficient inhibitory control. Failure of this deactivation correlated with white matter damage in tracts connecting the salience network nodes. Menon and Uddin (2010) therefore propose that the salience network organizes DMN and ECN activity to account for an appropriate response to salient stimuli (Menon and Uddin, 2010). Disrupted salience network functioning has also been associated with a multitude of other cognitive disorders, among which are schizophrenia (EllisonWright and Bullmore, 2010), psychosis (Palaniyappan and Liddle, 2012), bipolar disorder (Ellison-Wright and Bullmore, 2010), autism (Menon and Uddin, 2010), and frontotemporal dementia (Seeley, 2008, 2010). Further research should elucidate how the disruptions in DMN and ECN functioning during propofol anesthesia relate to disrupted salience network functioning.

\section{Thalamus}

Seed analysis from the thalamus showed decreases in connectivity between thalamus and regions overlapping with the $\mathrm{DMN}$ and ECN, similar to those found in the study of 


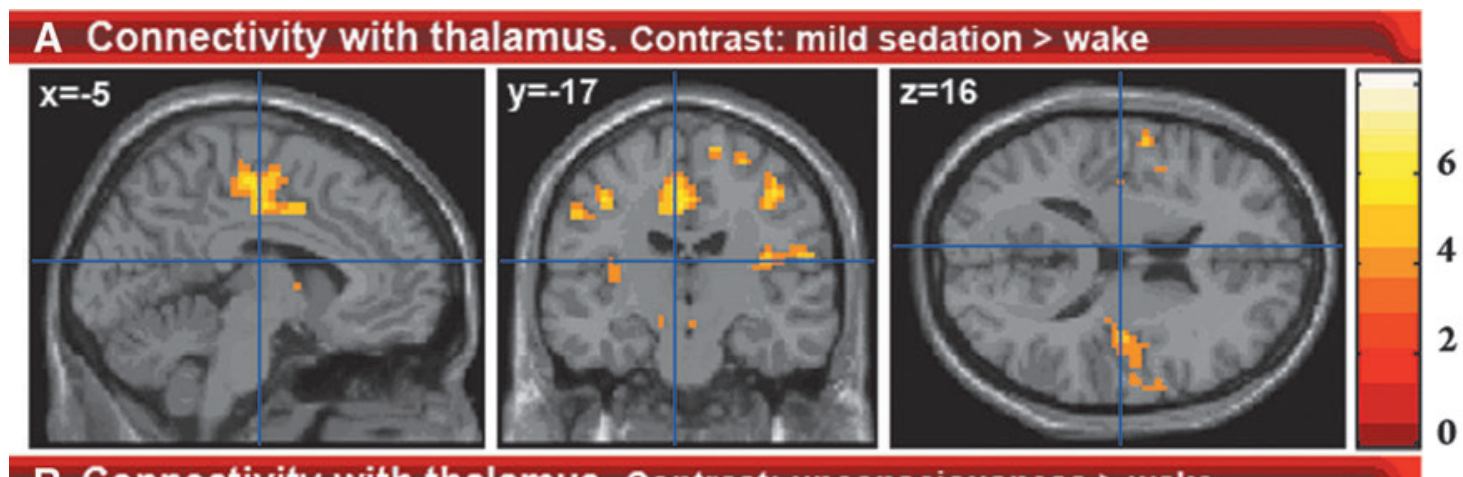

B Connectivity with thalamus. Contrast: unconsciousness $>$ wake
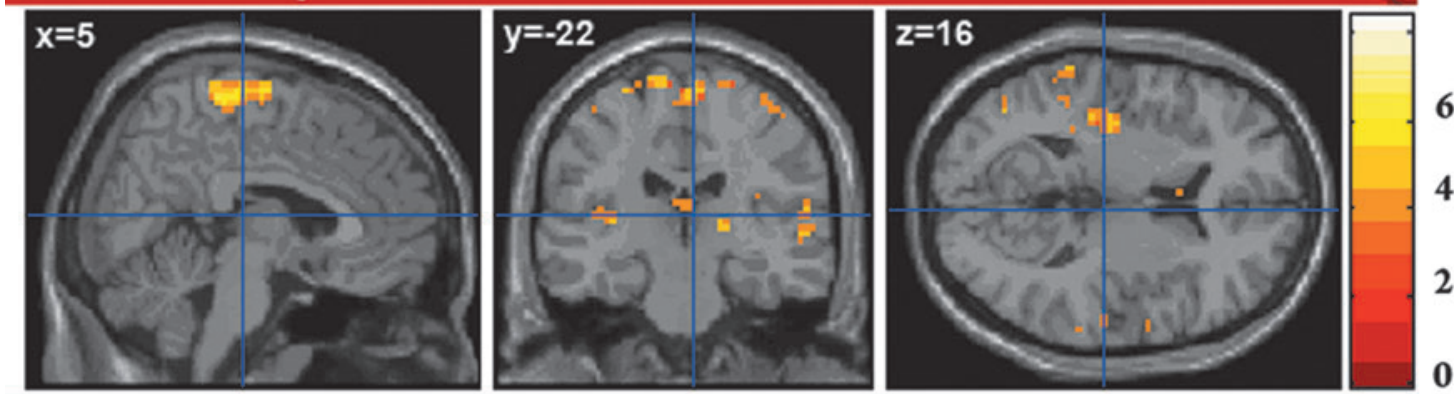

C Connectivity with thalamus. Contrast: mild sedation > unconsciousness
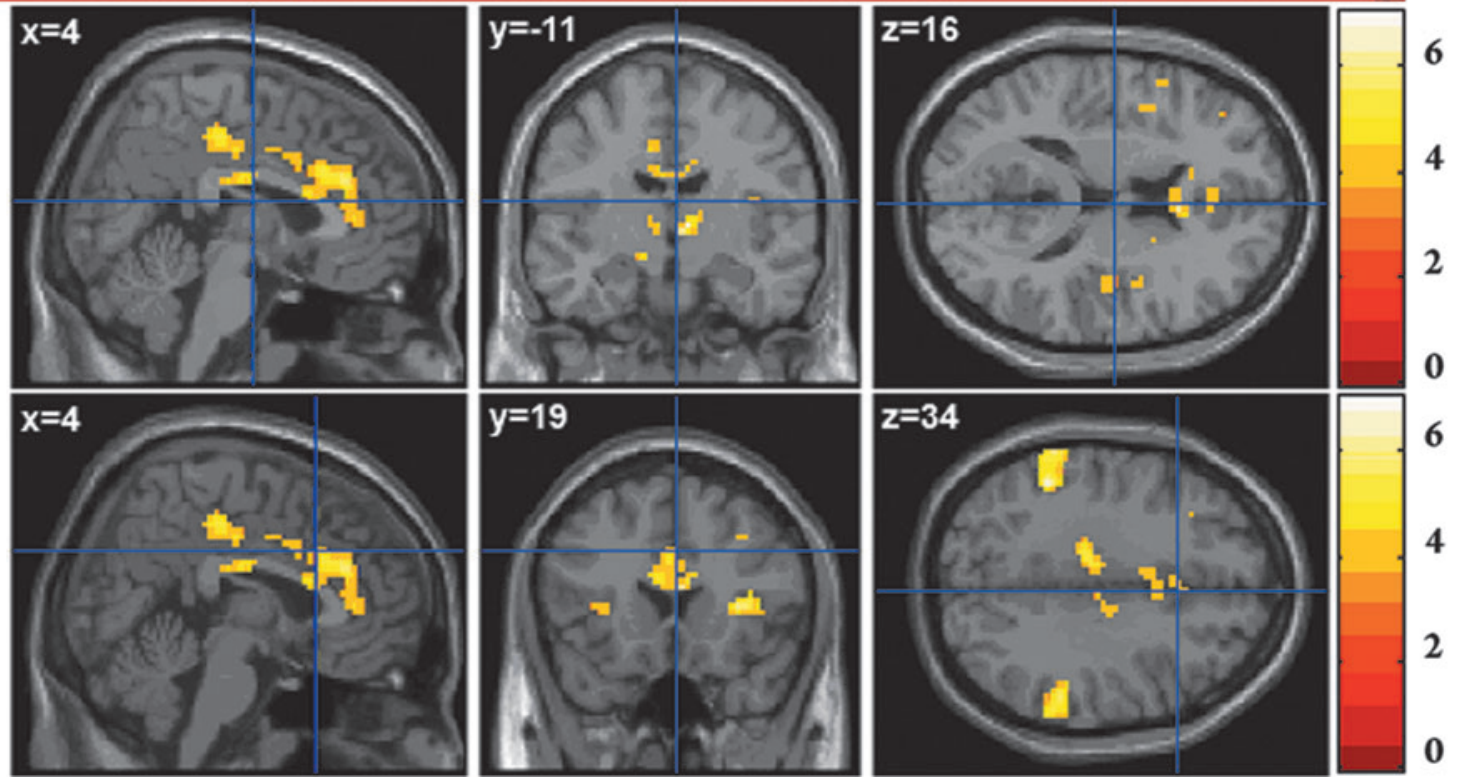

FIG. 3. Changes in connectivity with the thalamus. (A) Mild sedation $>$ wake, showing an increase in connectivity to the bilateral insulae and sensorimotor cortex; (B) unconsciousness $>$ wake, also showing increased thalamus-insula and thalamus-motor cortex connectivity; (C) mild sedation > unconsciousness. From mild sedation to unconsciousness, a decrease in connectivity between thalamus and inferior parietal lobules and salience RSN regions (insular and anterior cingulate cortices) is observed ( $p<0.001$, see Supplementary Material S4 for whole brain and small volume corrections).

Boveroux et al. (2010), as well as the salience RSN. Therefore, unconsciousness seems to correlate with disintegration of all three major higher-order networks, as well as their connectivity with the thalamus. Using positron emission tomography, thalamic metabolism has been shown to decrease significantly during anesthesia-induced unconsciousness (Fiset et al., 1999). Furthermore, a model has been suggested in which the thalamus orchestrates the commonly observed increased and coherent alpha frequency activity in the frontal cortex during propofol-induced unconsciousness (Ching et al., 2010; Cimenser et al., 2011). The authors suggest that this steady thalamic alpha rhythm could impede conduction and thus responsiveness to external stimuli. The fact that such a relatively active thalamus during unconsciousness has not been described for positron emission tomography and fMRI experiments could be because the signals obtained with these imaging methods are thought to be mostly associated with high-frequency activity, rather than alpha activity (Lachaux et al., 2007). It is not unlikely that links might exist between increased frontal cortex alpha activity and the 


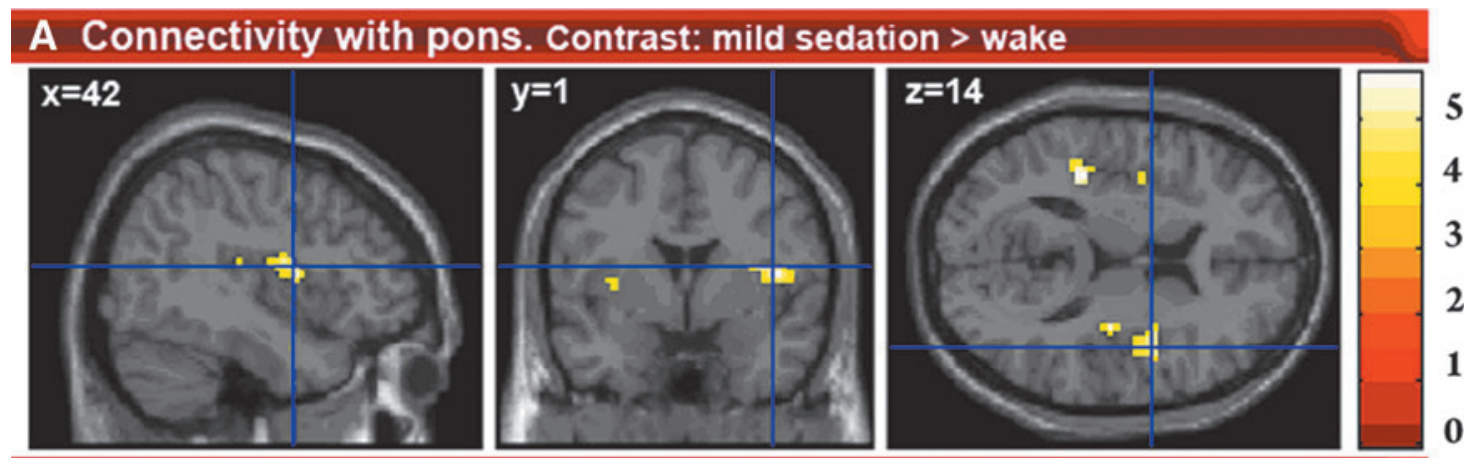

B Connectivity with pons. Contrast: unconsciousness > wake
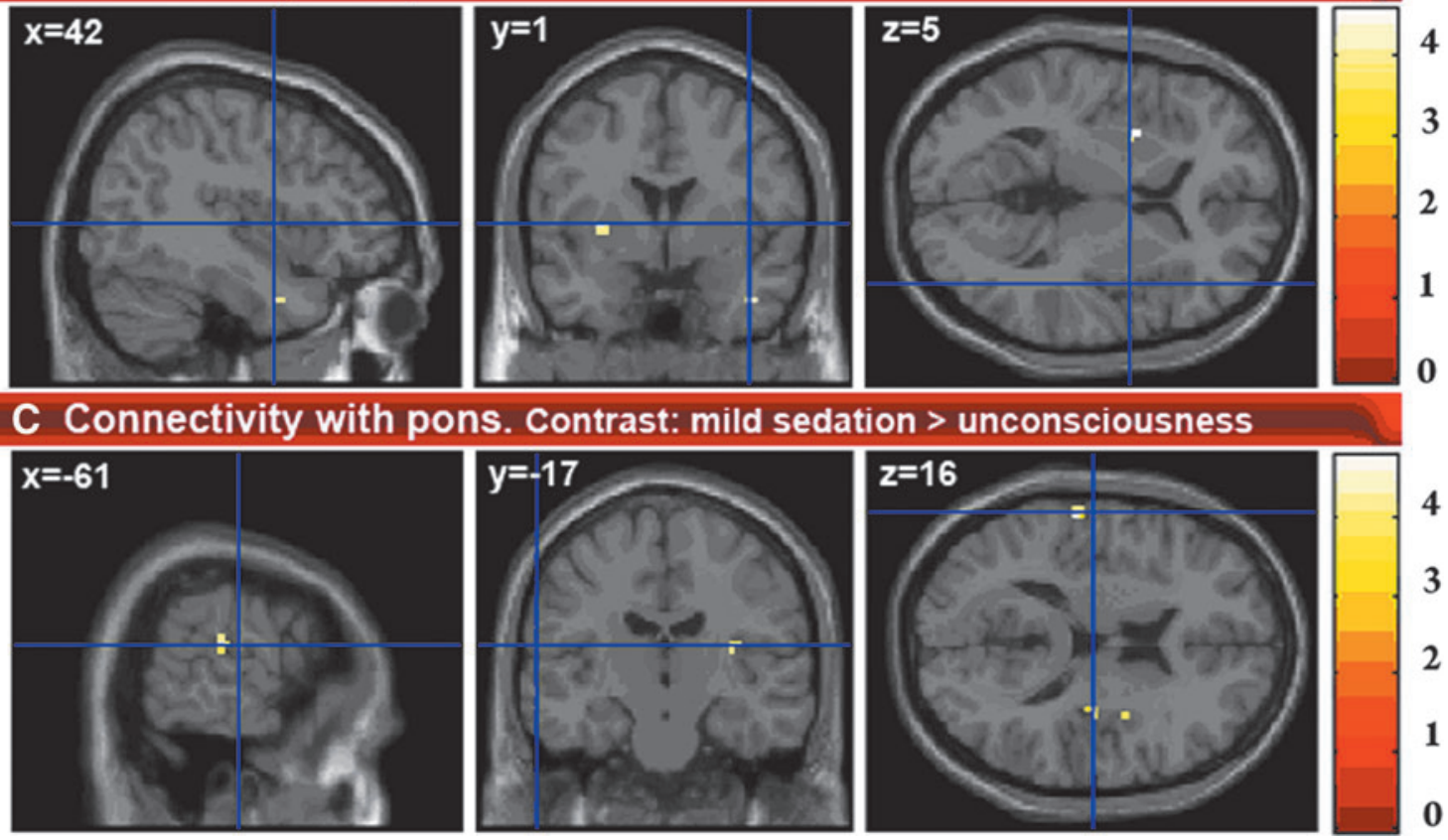

FIG. 4. Changes in connectivity with the PTA. (A) Mild sedation $>$ wake, showing increased connectivity of the PTA with bilateral insulae; (B) unconsciousness > wake, showing an increase in PTA-putamen connectivity; (C) mild sedation > unconsciousness. From mild sedation to unconsciousness, the increased PTA-insula connectivity found with mild sedation decreases to a preanesthesia level ( $p<0.001$, see Supplementary Material S4 for whole brain and small volume corrections).

disconnection of frontal cortex components of the DMN and salience network described in this paper. Indeed, new evidence supports the view that alpha activity in a certain brain area might be associated with inhibition in that area (Jensen and Mazaheri, 2010). In the awake brain, alpha activity is thought to serve maintenance and change of attentional focus by inhibition of distractors (Ben-Simon et al., 2013; Foxe and Snyder, 2011; Hanslmayr et al., 2011; Mathewson et al., 2011). In the unconscious brain, alpha activity might reflect inhibition of perception (Ching et al., 2010).

We observed increased connectivity between thalamus and both sensorimotor and auditory/insular cortices during mild sedation (Fig. 5A). Although increased thalamic connectivity with these regions has previously been observed during mild sevoflurane sedation (Martuzzi et al., 2010), to our knowledge, no previous resting state fMRI reports exist on the persistence of such increased connectivity during unconsciousness (Fig. 5B). One possible partial explanation for this connectivity increase might be that brain disturbance by propofol favors functional connectivity between brain regions that make up an above average contribution to the functional and possibly structural connective repertoire of the central thalamus. This is the case for the sensorimotor cortices (Fair et al., 2010). However, further research should elucidate the origin of this increased connectivity.

\section{Brainstem}

The brainstem contains a major part of the ascending reticular arousal system (de Lecea et al., 2012; Saper et al., 2005). Using our ICA of the DMN, we detected involvement of the mesencephalon within the DMN with recovery from unconsciousness. With seed analysis, we found propofol-induced decreased connectivity between DMN regions, especially the posterior cingulate cortex/precuneus, and both PTA and mesencephalon. The posterior cingulate cortex/precuneus is one of the most active regions in the human brain during wakefulness, and diminished activity here has been associated with loss of consciousness due to anesthesia and disorders of consciousness (Laureys, 2008; Xie et al., 2011). The importance of 
the PTA in the ascending reticular arousal system is well illustrated in the case of brainstem coma, a state in which there is a total absence of brain arousal and awareness (Laureys, 2008), where bilateral damage in the PTA alone can induce coma (Parvizi and Damasio, 2003). Recovery of brainstem functioning during recovery from coma shows similarities with recovery from anesthesia (Brown et al., 2010).

The mesencephalon has been shown to havesolid connections with the posterior cingulate cortex/precuneus and anterior cingulate cortex in a multitude of studies and is considered to be a key region in pain saliency processing (Linnman et al., 2012). Moreover, two nuclei in the mesencephalon, the periaquaductal gray matter and the parabrachial nucleus, are thought to have a prominent role in modulating global brain activity. Projections have been found from these nuclei to the brain arousal areas of the reticular nuclei and basal forebrain, as well as intralaminar thalamic nuclei (Parvizi and Damasio, 2001).

Considering the brain arousal modulating capacities of the pontine and mesencephalic nuclei, their decreased connectivity with DMN regions during unconsciousness and the increasing connectivity between DMN and mesencephalon during recovery suggest brainstem-driven changes in brain arousal to partly underlie propofol-induced DMN connectivity decreases. These DMN decreases include disconnection of

\section{A Changes in connectivity during mild sedation}

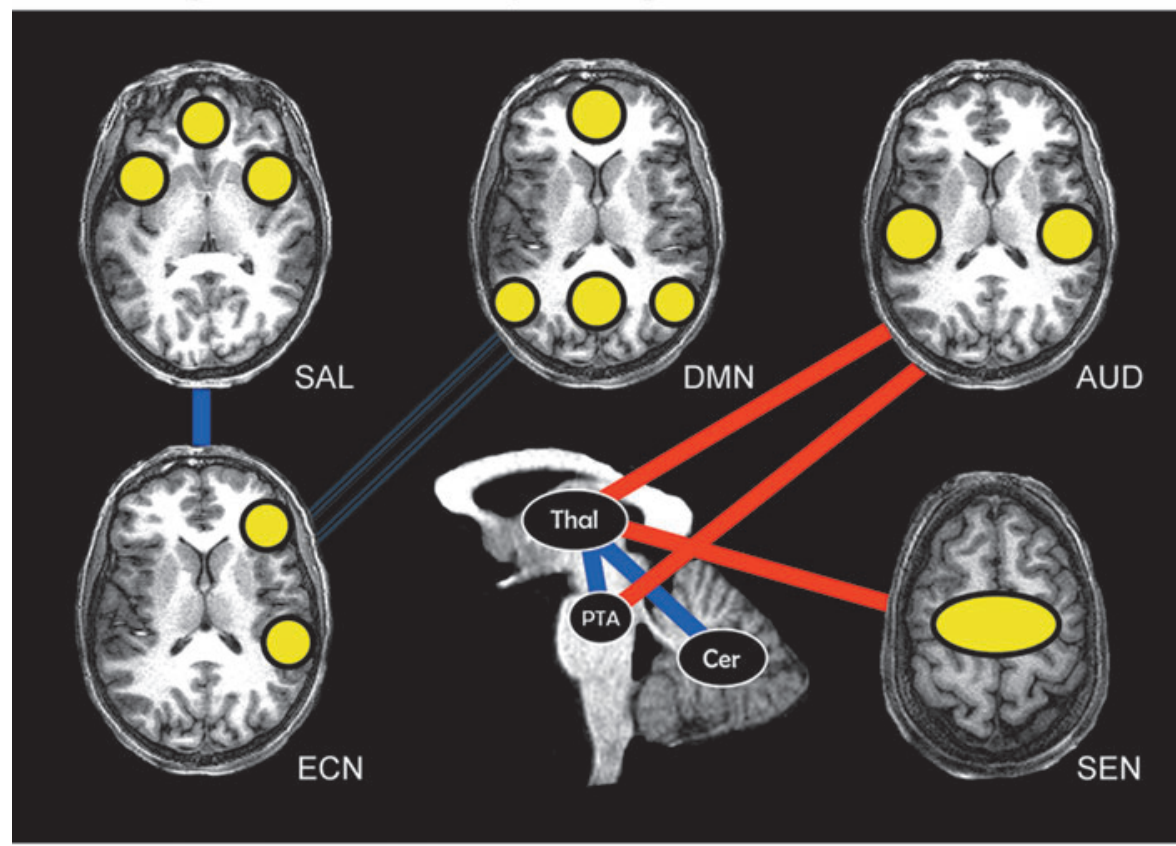

B Changes in connectivity during unconsciousness

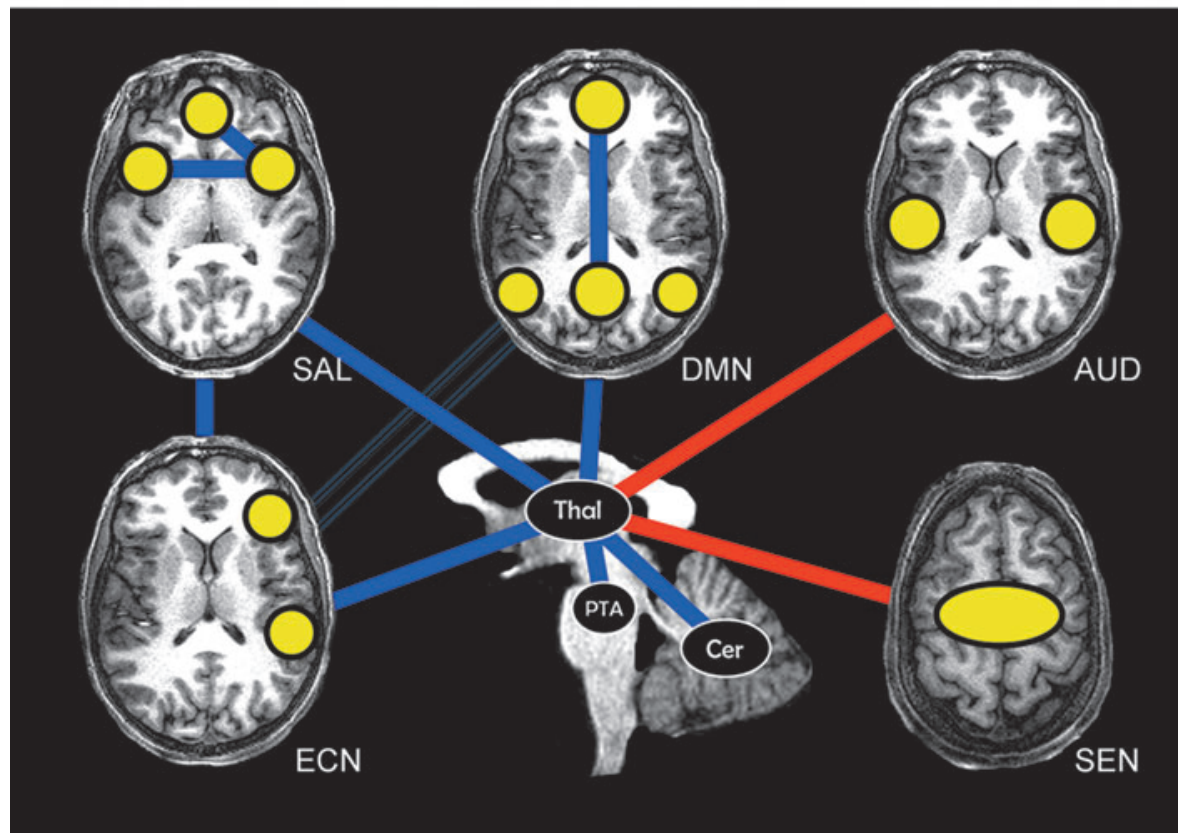

FIG. 5. Summary of propofolinduced connectivity changes. (A) Connectivity increases (red lines) and decreases (blue lines), as well as decreased anticorrelation (thin blue double lines) during mild sedation as compared to wakefulness. (B) Connectivity increases (red lines) and decreases (blue lines), as well as decreased anticorrelation (thin blue double lines) during unconsciousness as compared to wakefulness. SAL, salience network; AUD, auditory network/ insular cortex; ECN, external control network; Thal, thalamus; Cer, cerebellum; SEN, sensorimotor cortex. Yellow blobs represent network nodes. Lines represent resting state connectivity between regions and/or networks. 
the frontal cortex and the hippocampus. A decreased connectivity between thalamus and PTA was also found; two pivotal entities in brain arousal that are known to work closely together (Saper et al., 2005). Decreased connectivity between them could potentially also represent a change in coordination of brain arousal.

We observed increased connectivity between PTA and insular cortices with mild sedation. These PTA connectivity changes have not been previously described and further research should indicate whether they could potentially underlie changes in brain processing associated with the insulae, such as auditory processing, salience detection, and self-perception (Menon and Uddin, 2010; Modinos et al., 2009; Saper et al., 2005). No increased PTA connectivity with the insular cortex was found during unconsciousness as compared to wakefulness. This suggests a nonlinear behavior during decreasing levels of consciousness, with increased connectivity during mild sedation but not unconsciousness. The reason for the absence of this increased connectivity between PTA and insulae during unconsciousness might be related to the state of general depression in the brain during unconsciousness, impeding salience detection and execution of behavior.

\section{Methodological considerations}

In our study, we used both ICA- and seed-based methods to try to examine connectivity decreases and increases during mild sedation and unconsciousness. For this, we used resting state fMRI, which assumes that low frequency changes in blood oxygenation reflect neuronal activity. ICA is an established data-driven method that is an adequate tool for exploring data and discriminating neuronal activity from artifact (Hyvarinen et al., 2001). It relies on the method of maximum discrimination of brain activity frequencies within a dataset given a certain number of components. However, potential bias might occur during the component selection step, as well as from the assumption that in every consciousness condition, remnants of the RSN of interest (e.g., DMN) are still present. The seed-based technique does not have these kinds of biases. However, given that proper preprocessing has been conducted, seed-based techniques might still suffer from the bias of ROI selection. Seed-based analysis shows the total connective repertoire of a chosen ROI. It looks at the degree of similarity of brain activity with all frequency patterns found in the ROI. Therefore, the techniques might be considered to be complementary, although comparing findings obtained using ICA with those gathered with seed-based methods requires some caution, especially when using different software packages for each method. However, we consider our results as being robust, as both the ICA- and seed-based methods found increased connectivity between thalamus and insular/auditory areas during mild sedation. Inspection of component fingerprints (De Martino et al., 2007) showed that it is possible to reliably find and study RSNs during propofol-induced unconsciousness.

Anesthesia could potentially affect the number of RSNs present in the brain. If this number changes, and we still only divide into 30 independent components with ICA, possibly RSNs are grouped together that would normally not be incorporated in the same independent component, or RSN regions could stop being associated with a certain RSN and become associated with another RSN in another independent component. A seem- ing disconnection or connectivity increase may thus be the result of a changing number of discriminable RSNs available in the brain. However, previously published research using seed-based methodology also showed propofol-induced disconnection of the frontal part of the DMN (Boveroux et al., 2010). Increased connectivity in the auditory RSN seen during mild sedation with ICA was also found with our seed-based method. Therefore, although it is imaginable that propofol might confound by changing discriminability of RSNs, we do not expect that this will affect our results greatly.

A possible explanation for the increased connectivity between thalamus and primary sensorimotor areas might be increased body movement during propofol sedation. Although we did not observe significant head motion, we did not perform electromyography to exclude significant body movement. However, a recent high-density electroencephalography study with propofol (Murphy et al., 2011) examined subjects at Ramsey 3 (mild sedation) and 5 (unconsciousness) levels of sedation; comparable to the sedation levels used in our study. Using electromyography, they found no significantly increased body movement during sedation stages. However, they report an increase in spindle (12$15 \mathrm{~Hz})$ and beta $(15-25 \mathrm{~Hz})$ power during mild sedation, while increased gamma $(25-40 \mathrm{~Hz})$ power was observed in both mild sedation and unconsciousness. The authors excluded the possibility that these increases originated from ocular or muscular sources. Using electroencephalography source reconstruction, another report showed increased thalamic excitability during mild sedation and unconsciousness (Boly et al., 2012).

A further point of caution is the discrimination between connectivity and correlated activity. Although the central thalamic nuclei are often regarded as part of a vital brain arousal pathway with a broad connective range (Brown et al., 2010; Schiff, 2008), our findings cannot single out the possibility that increased connectivity with sensorimotor/insular regions might rather be a form of coincidental correlation. An in vivo cell recording study in rats with lesions in the central lateral intralaminar nucleus, destroying thalamic afferent connections, showed that brain arousal of local cortical networks might not be associated with thalamic afferent input (Constantinople and Bruno, 2011). Synchronous activity of thalamus and sensorimotor/insular cortices could therefore also be a result of both regions receiving similar input from another brain region, or being connected via that other region. However, other studies have shown significant decreases in brain arousal associated with central thalamic injury and significant deafferentation of its neurons due to diffuse brain insults in humans (Schiff and Plum, 2000; Shah and Schiff, 2010). Furthermore, sevoflurane-induced unconsciousness has been reversed by microinjection of nicotine, a cholinergic agonist, into the rat central thalamus (Alkire et al., 2007). These results emphasize the close direct relationship of the thalamus with the cortex, suggesting propofol influences direct connectivity between thalamus and cortical regions. It is possible that complementary arousal pathways provide an indirect connection between thalamus and cortical regions. An example would be the part of the ascending arousal pathway that does not run through the thalamus, but is influenced by it (Saper et al., 2005). Further research should elucidate the exact role of thalamic nuclei in cortical excitation during states of altered consciousness.

Another possible reason for carefulness when interpreting fMRI data might be the potential influence of $\mathrm{pCO} 2$ levels on 
the BOLD signal. However, it has been shown that pCO2 levels do not seem to change the BOLD response to neuronal activity (Birn et al., 2006; Wise et al., 2004). Furthermore, for our analysis, we are interested in correlations rather than specific regional effects and are therefore confident $\mathrm{pCO} 2$ levels do not significantly influence our results (Corfield et al., 2001).

\section{Conclusion}

Our study shows that propofol-induced mild sedation and unconsciousness is associated with decreased connectivity within the salience network, in addition to previously reported DMN and ECN breakdown. Thalamic connectivity with these three higher-order RSNs was also found to be reduced. Furthermore, brainstem connectivity with DMN-related regions decreased. In contrast, connectivity increases were observed between the thalamus and sensorimotor/insular/auditory cortices, as well as between the PTA and insular/auditory cortices. These findings give insight into how connectivity changes in DMN and ECN internal and external awareness networks might be related to salience network integrity and arousal components located in the thalamus and brainstem. It emphasizes the necessity of examining resting state connectivity of brain regions associated with both brain arousal and awareness together to make inferences upon mechanisms responsible for loss of consciousness.

\section{Funding and Acknowledgments}

This research was funded by the Belgian National Funds for Scientific Research (Brussels, Belgium), the European Commission (Brussels, Belgium), the James McDonnell Foundation (Saint Louis, Missouri, USA), the Mind Science Foundation (San Antonio, Texas, USA), the French Speaking Community Concerted Research Action (ARC, 06/11-340, Brussels, Belgium), the Fondation Médicale Reine Elisabeth (Brussels, Belgium), the University of Liège (Liège, Belgium) and the University Hospital of Liège (Liège, Belgium).

\section{Author Disclosure Statement}

No competing financial interests exist.

\section{References}

Abulafia R, Zalkind V, Devor M. 2009. Cerebral activity during the anesthesia-like state induced by mesopontine microinjection of pentobarbital. J Neurosci 29:7053-7064.

Alkire MT, McReynolds JR, Hahn EL, Trivedi AN. 2007. Thalamic microinjection of nicotine reverses sevoflurane-induced loss of righting reflex in the rat. Anesthesiology 107:264-272.

Angeles Fernandez-Gil M, Palacios-Bote R, Leo-Barahona M, Mora-Encinas JP. 2010. Anatomy of the brainstem: a gaze into the stem of life. Semin Ultrasound CT MR 31:196-219.

Aron AR, Robbins TW, Poldrack RA. 2004. Inhibition and the right inferior frontal cortex. Trends Cogn Sci 8:170-177.

Beckmann CF, DeLuca M, Devlin JT, Smith SM. 2005. Investigations into resting-state connectivity using independent component analysis. Philos Trans R Soc Lond B Biol Sci 360: 1001-1013.

Ben-Simon E, Podlipsky I, Okon-Singer H, Gruberger M, Cvetkovic D, Intrator N, Hendler T. 2013. The dark side of the alpha rhythm: fMRI evidence for induced alpha modulation during complete darkness. Eur J Neurosci 37:795-803.
Birn RM, Diamond JB, Smith MA, Bandettini PA. 2006. Separating respiratory-variation-related fluctuations from neuronal-activity-related fluctuations in fMRI. Neuroimage 31:1536-1548.

Boly M, Moran R, Murphy M, Boveroux P, Bruno MA, Noirhomme Q, Ledoux D, Bonhomme V, Brichant JF, Tononi G, Laureys S, Friston K. 2012. Connectivity changes underlying spectral EEG changes during propofol-induced loss of consciousness. J Neurosci 32:7082-7090.

Boly M, Tshibanda L, Vanhaudenhuyse A, Noirhomme Q, Schnakers C, Ledoux D, Boveroux P, Garweg C, Lambermont B, Phillips C, Luxen A, Moonen G, Bassetti C, Maquet P, Laureys S. 2009. Functional connectivity in the default network during resting state is preserved in a vegetative but not in a brain dead patient. Hum Brain Mapp 30:2393-2400.

Bonnelle V, Ham TE, Leech R, Kinnunen KM, Mehta MA, Greenwood RJ, Sharp DJ. 2012. Salience network integrity predicts default mode network function after traumatic brain injury. Proc Natl Acad Sci U S A 109:4690-4695.

Boveroux P, Vanhaudenhuyse A, Bruno MA, Noirhomme Q, Lauwick S, Luxen A, Degueldre C, Plenevaux A, Schnakers C, Phillips C, Brichant JF, Bonhomme V, Maquet P, Greicius MD, Laureys S, Boly M. 2010. Breakdown of within- and between-network resting state functional magnetic resonance imaging connectivity during propofol-induced loss of consciousness. Anesthesiology 113:1038-1053.

Brown EN, Lydic R, Schiff ND. 2010. General anesthesia, sleep, and coma. N Engl J Med 363:2638-2650.

Brown EN, Purdon PL, Van Dort CJ. 2011. General anesthesia and altered states of arousal: a systems neuroscience analysis. Annu Rev Neurosci 34:601-628.

Ching S, Cimenser A, Purdon PL, Brown EN, Kopell NJ. 2010. Thalamocortical model for a propofol-induced alpha-rhythm associated with loss of consciousness. Proc Natl Acad Sci U S A 107:22665-22670.

Cimenser A, Purdon PL, Pierce ET, Walsh JL, Salazar-Gomez AF, Harrell PG, Tavares-Stoeckel C, Habeeb K, Brown EN. 2011. Tracking brain states under general anesthesia by using global coherence analysis. Proc Natl Acad Sci U S A 108: 8832-8837.

Constantinople CM, Bruno RM. 2011 Effects and mechanisms of wakefulness on local cortical networks. Neuron 69:1061-1068.

Corfield DR, Murphy K, Josephs O, Adams L, Turner R. 2001. Does hypercapnia-induced cerebral vasodilation modulate the hemodynamic response to neural activation? Neuroimage 13:1207-1211.

Damoiseaux JS, Rombouts SA, Barkhof F, Scheltens P, Stam CJ, Smith SM, Beckmann CF. 2006. Consistent resting-state networks across healthy subjects. Proc Natl Acad Sci U S A 103:13848-13853.

de Lecea L, Carter ME, Adamantidis A. 2012. Shining light on wakefulness and arousal. Biol Psychiatry 71:1046-1052.

De Martino F, Gentile F, Esposito F, Balsi M, Di Salle F, Goebel R, Formisano E. 2007. Classification of fMRI independent components using IC-fingerprints and support vector machine classifiers. NeuroImage 34:177-194.

Demertzi A, Soddu A, Faymonville ME, Bahri MA, Gosseries O, Vanhaudenhuyse A, Phillips C, Maquet P, Noirhomme Q, Luxen A, Laureys S. 2011. Hypnotic modulation of resting state fMRI default mode and extrinsic network connectivity. Prog Brain Res 193:309-322.

Devor M, Zalkind V. 2001. Reversible analgesia, atonia, and loss of consciousness on bilateral intracerebral microinjection of pentobarbital. Pain 94:101-112. 
Duann JR, Ide JS, Luo X, Li CS. 2009. Functional connectivity delineates distinct roles of the inferior frontal cortex and presupplementary motor area in stop signal inhibition. J Neurosci 29:10171-10179.

Ellison-Wright I, Bullmore E. 2010. Anatomy of bipolar disorder and schizophrenia: a meta-analysis. Schizophr Res 117:1-12.

Fair DA, Bathula D, Mills KL, Dias TG, Blythe MS, Zhang D, Snyder AZ, Raichle ME, Stevens AA, Nigg JT, Nagel BJ. 2010. Maturing thalamocortical functional connectivity across development. Front Syst Neurosci 4:10.

Fair DA, Cohen AL, Dosenbach NU, Church JA, Miezin FM, Barch DM, Raichle ME, Petersen SE, Schlaggar BL. 2008. The maturing architecture of the brain's default network. Proc Natl Acad Sci U S A 105:4028-4032.

Fiset P, Paus T, Daloze T, Plourde G, Meuret P, Bonhomme V, Hajj-Ali N, Backman SB, Evans AC. 1999. Brain mechanisms of propofol-induced loss of consciousness in humans: a positron emission tomographic study. J Neurosci 19:5506-5513.

Fox MD, Snyder AZ, Vincent JL, Corbetta M, Van Essen DC, Raichle ME. 2005. The human brain is intrinsically organized into dynamic, anticorrelated functional networks. Proc Natl Acad Sci U S A 102:9673-9678.

Foxe JJ, Snyder AC. 2011. The role of alpha-band brain oscillations as a sensory suppression mechanism during selective attention. Front Psychol 2:154.

Giacino J, Fins JJ, Machado A, Schiff ND. 2012. Central thalamic deep brain stimulation to promote recovery from chronic posttraumatic minimally conscious state: challenges and opportunities. Neuromodulation 15:339-349.

Greicius MD, Kiviniemi V, Tervonen O, Vainionpaa V, Alahuhta S, Reiss AL, Menon V. 2008. Persistent default-mode network connectivity during light sedation. Hum Brain Mapp 29:839-847.

Guldenmund P, Vanhaudenhuyse A, Boly M, Laureys S, Soddu A. 2012. A default mode of brain function in altered states of consciousness. Arch Ital Biol 150:107-121.

Hanslmayr S, Gross J, Klimesch W, Shapiro KL. 2011. The role of alpha oscillations in temporal attention. Brain Res Rev 67: 331-343.

Horovitz SG, Braun AR, Carr WS, Picchioni D, Balkin TJ, Fukunaga M, Duyn JH. 2009. Decoupling of the brain's default mode network during deep sleep. Proc Natl Acad Sci U S A 106:11376-11381.

Hyvarinen A, Karhunen J, Oja E. 2001. Independent Component Analysis. John Wiley \& Sons.

Jensen O, Mazaheri A. 2010. Shaping functional architecture by oscillatory alpha activity: gating by inhibition. Front Hum Neurosci 4:186.

Lachaux JP, Fonlupt P, Kahane P, Minotti L, Hoffmann D, Bertrand O, Baciu M. 2007. Relationship between task-related gamma oscillations and BOLD signal: new insights from combined fMRI and intracranial EEG. Hum Brain Mapp 28:1368-1375.

Laureys S, Boly M. 2008. The changing spectrum of coma. Nat Clin Pract Neurol 4:544-546.

Lieberman MD, Cunningham WA. 2009. Type I and Type II error concerns in fMRI research: re-balancing the scale. Soc Cogn Affect Neurosci 4:423-428.

Linnman C, Moulton EA, Barmettler G, Becerra L, Borsook D. 2012. Neuroimaging of the periaqueductal gray: state of the field. Neuroimage 60:505-522.

Martuzzi R, Ramani R, Qiu M, Rajeevan N, Constable RT. 2010. Functional connectivity and alterations in baseline brain state in humans. Neuroimage 49:823-834.

Mathewson KE, Lleras A, Beck DM, Fabiani M, Ro T, Gratton G. 2011. Pulsed out of awareness: EEG alpha oscillations repre- sent a pulsed-inhibition of ongoing cortical processing. Front Psychol 2:99.

Maudoux A, Lefebvre P, Cabay JE, Demertzi A, Vanhaudenhuyse A, Laureys S, Soddu A. 2012. Connectivity graph analysis of the auditory resting state network in tinnitus. Brain Res 1485:10-21.

McKeown MJ, Makeig S, Brown GG, Jung TP, Kindermann SS, Bell AJ, Sejnowski TJ. 1998. Analysis of fMRI data by blind separation into independent spatial components. Hum Brain Mapp 6:160-188.

Menon V, Uddin LQ. 2010. Saliency, switching, attention and control: a network model of insula function. Brain Struct Funct 214:655-667.

Modinos G, Ormel J, Aleman A. 2009. Activation of anterior insula during self-reflection. PLoS One 4:e4618.

Murphy M, Bruno MA, Riedner BA, Boveroux P, Noirhomme Q, Landsness EC, Brichant JF, Phillips C, Massimini M, Laureys S, Tononi G, Boly M. 2011. Propofol anesthesia and sleep: a high-density EEG study. Sleep 34:283-291A.

Olson DM, Thoyre SM, Auyong DB. 2007. Perspectives on sedation assessment in critical care. AACN Adv Crit Care 18: 380-395.

Palaniyappan L, Liddle PF. 2012. Does the salience network play a cardinal role in psychosis? An emerging hypothesis of insular dysfunction. J Psychiatry Neurosci 37:17-27.

Parvizi J, Damasio A. 2001. Consciousness and the brainstem. Cognition 79:135-160.

Parvizi J, Damasio AR. 2003. Neuroanatomical correlates of brainstem coma. Brain 126:1524-1536.

Pinault D. 2011. Dysfunctional thalamus-related networks in schizophrenia. Schizophr Bull 37:238-243.

Saper CB, Cano G, Scammell TE. 2005. Homeostatic, circadian, and emotional regulation of sleep. J Comp Neurol 493:92-98.

Schiff ND. 2008. Central thalamic contributions to arousal regulation and neurological disorders of consciousness. Ann N Y Acad Sci 1129:105-118.

Schiff ND, Fins JJ. 2007. Deep brain stimulation and cognition: moving from animal to patient. Curr Opin Neurol 20:638-642.

Schiff ND, Plum F. 2000. The role of arousal and "gating" systems in the neurology of impaired consciousness. J Clin Neurophysiol 17:438-452.

Schrouff J, Perlbarg V, Boly M, Marrelec G, Boveroux P, Vanhaudenhuyse A, Bruno MA, Laureys S, Phillips C, Pelegrini-Issac M, Maquet P, Benali H. 2011. Brain functional integration decreases during propofol-induced loss of consciousness. Neuroimage 57:198-205.

Seeley WW. 2008. Selective functional, regional, and neuronal vulnerability in frontotemporal dementia. Curr Opin Neurol 21:701-707.

Seeley WW. 2010. Anterior insula degeneration in frontotemporal dementia. Brain Struct Funct 214:465-475.

Seeley WW, Menon V, Schatzberg AF, Keller J, Glover GH, Kenna H, Reiss AL, Greicius MD. 2007. Dissociable intrinsic connectivity networks for salience processing and executive control. J Neurosci 27:2349-2356.

Shah SA, Schiff ND. 2010. Central thalamic deep brain stimulation for cognitive neuromodulation-a review of proposed mechanisms and investigational studies. Eur J Neurosci 32:1135-1144.

Soddu A, Vanhaudenhuyse A, Bahri MA, Bruno MA, Boly M, Demertzi A, Tshibanda JF, Phillips C, Stanziano M, OvadiaCaro S, Nir Y, Maquet P, Papa M, Malach R, Laureys S, Noirhomme Q. 2012. Identifying the default-mode component in spatial IC analyses of patients with disorders of consciousness. Hum Brain Mapp 33:778-796. 
Tang L, Ge Y, Sodickson DK, Miles L, Zhou Y, Reaume J, Grossman RI. 2011. Thalamic resting-state functional networks: disruption in patients with mild traumatic brain injury. Radiology 260:831-840.

Vanhaudenhuyse A, Demertzi A, Schabus M, Noirhomme Q, BredartS, Boly M, Phillips C, Soddu A, Luxen A, Moonen G, Laureys S. 2011. Two distinct neuronal networks mediate the awareness of environment and of self. J Cogn Neurosci 23:570-578.

Vanhaudenhuyse A, Noirhomme Q, Tshibanda LJ, Bruno MA, Boveroux P, Schnakers C, Soddu A, Perlbarg V, Ledoux D, Brichant JF, Moonen G, Maquet P, Greicius MD, Laureys S, Boly M. 2009. Default network connectivity reflects the level of consciousness in non-communicative brain-damaged patients. Brain 133:161-171.

Wang DS, Orser BA. 2011. Inhibition of learning and memory by general anesthetics. Can J Anaesth 58:167-177.

Wise RG, Ide K, Poulin MJ, Tracey I. 2004. Resting fluctuations in arterial carbon dioxide induce significant low frequency variations in BOLD signal. Neuroimage 21:1652-1664.

Xie G, Deschamps A, Backman SB, Fiset P, Chartrand D, Dagher A, Plourde G. 2011. Critical involvement of the thalamus and precuneus during restoration of consciousness with physostigmine in humans during propofol anaesthesia: a positron emission tomography study. Br J Anaesth 106:548-557.
Address correspondence to: Pieter Guldenmund Coma Science Group Cyclotron Research Center

University of Liège B30, Allée du 6 août Sart Tilman 4000 Liège Belgium

E-mail: guldenmund@hotmail.com

Steven Laureys Coma Science Group Cyclotron Research Center University of Liège B30, Allée du 6 août

Sart Tilman 4000 Liège Belgium

E-mail: steven.laureys@ulg.ac.be 